\title{
Les Services Système : l'expérience de RTE
}

\author{
T. Margotin, P. Juston, M.P. Houry \\ Réseau de Transport d'Electricité (RTE) \\ Département Méthodes et Appui \\ 9 rue de la Porte de Buc \\ Versailles, France
}

\begin{abstract}
Résumé- L'objet de cette présentation est de faire part de l'expérience de RTE en matière de Services Système. Après une description des contextes réglementaire, contractuel et technique, sont présentées les caractéristiques de l'outil de Contrôle de Performances, qui permet de vérifier que les unités de production respectent leurs engagements contractuels vis à vis des réglages de fréquence et de tension.
\end{abstract}

\section{INTRODUCTION}

RTE, garant de la sûreté du système électrique français, se doit de veiller à la disponibilité des Services Système, services dont tous les utilisateurs du Réseau Public de Transport bénéficient, quel que soit le niveau de tension auquel ils sont raccordés. Les Services Système sont réalisés à l'aide du réglage de la fréquence et du réglage de la tension. Ce sont principalement les producteurs qui contribuent à ces réglages en mettant à disposition les capacités de régulation de leurs installations de production.

Les enjeux pour le système électrique et les utilisateurs $\mathrm{du}$ réseau de transport sont importants. Les réglages de tension et de fréquence permettent de maintenir la tension et la fréquence dans des plages qui garantissent un niveau de sûreté suffisant et qui sont définies dans les contrats d'accès au réseau, de respecter les contraintes de fonctionnement des matériels et de limiter par leur efficacité les risques d'effondrement de réseau. L'analyse du blackout de 2003 aux Etats-Unis et au Canada a bien mis en exergue toute l'importance d'une gestion rigoureuse des réserves de réactif des moyens de production, [1].

Notre présentation est organisée de la manière suivante : après avoir décrit le contexte réglementaire et contractuel dans lequel s'effectue 1'acquisition des Services Système en France, nous expliciterons les caractéristiques techniques des réglages de tension et de fréquence français puis nous présenterons l'outil de Contrôle de Performances développé et mis en œuvre par RTE pour vérifier que les unités de production respectent leurs engagements contractuels en matière de Services Système.

\section{Elements DE CONTEXTE REgLEMENTAIRE ET CONTRACTUEL EN FranCE}

\section{A. Raccordement d'un nouveau moyen de production}

Pour être raccordée au Réseau Public de Transport, toute nouvelle installation de production doit disposer des capacités techniques lui permettant de contribuer aux Services Systèmes. Les décrets et arrêtés techniques [2], [3] fixent les exigences applicables : capacités constructives de réglage (réactif, réglage primaire, secondaire); participation ou non à certains types de réglage en fonction de la puissance de l'installation ou du niveau de tension; type de régulation; etc. Ces règles sont détaillées dans le Référentiel Technique publié par RTE, [4].

Quand une nouvelle centrale est raccordée au réseau, ces prescriptions sont déclinées dans une convention de raccordement, signée par le producteur et RTE. Des essais sur site avant mise en service sont réalisés afin de vérifier que l'installation satisfait les exigences vis à vis des réglages de tension et de fréquence.

\section{B. Acquisition des Services Système}

RTE acquiert les Services Système par des contrats bilatéraux avec les producteurs. Ainsi, au-delà de la convention de raccordement, un contrat spécifique «Services Système» est proposé aux producteurs. Ce contrat définit :

- les conditions financières, juridiques et techniques d'acquisition des Services Système (volumes de réserve prescrits, modalités de programmation,...);

- le mécanisme de contrôle des performances qui sera utilisé par RTE durant la vie du contrat pour vérifier le respect des engagements techniques contractualisés.

Le mécanisme de contrôle des performances est récent : il a été introduit en 2005. Il s'agit d'une vérification continue et a posteriori des performances de chaque unité de production, à partir de mesures disponibles au point de 
livraison. En cas d'écart par rapport aux engagements contractuels, le producteur s'engage à remettre son équipement en conformité selon un planning négocié avec RTE. Pendant cette période de remise en conformité et suivant l'importance de l'écart, la rémunération du producteur est réduite. Des pénalités sont appliquées si l'écart persiste au-delà de la période négociée.

\section{CARACTERIStiQues du Reglage De Tension}

La tension en un point du réseau dépend d'une part des forces électromotrices des moyens de production qui sont raccordés à proximité de l'endroit concerné, et d'autre part des chutes de tension dans les divers équipements du réseau tels que les lignes et les transformateurs.

La tension est naturellement fluctuante : elle est soumise à des variations lentes en fonction de l'évolution de la consommation; elle peut également varier rapidement suite à des fluctuations aléatoires de charges, à des changements de topologie de réseau, à des déclenchements d'ouvrages de production, etc.

La tension et la puissance réactive étant des grandeurs fortement liées, le réglage de la tension s'effectue par le contrôle de la puissance réactive. Celui-ci est réalisé d'une part à l'aide de moyens passifs de compensation de réactif (batteries de condensateurs, bobines d'inductance) installés sur le réseau et manœuvrables depuis les dispatchings, et d'autre part à l'aide des groupes de production pour un réglage rapide de la tension suite aux aléas d'exploitation.

\section{A. Réglage Primaire de la Tension}

Les alternateurs des installations de production doivent être équipés d'un régulateur primaire de tension, dispositif automatique qui asservit des grandeurs locales (la tension, ou la puissance réactive) à des valeurs de consigne en agissant sur la tension d'excitation de l'alternateur. L'action de ce régulateur est quasiment instantanée et permet de compenser les variations de tension rapides et aléatoires du réseau, tant que l'unité de production n'a pas atteint ses limites de réactif.

\section{B. Réglage Secondaire de la Tension}

Afin d'augmenter l'efficacité du réglage primaire de la tension et de coordonner l'action des alternateurs, certaines installations de production participent au réglage secondaire de la tension qui agit de façon automatique et coordonnée sur la consigne du régulateur primaire avec une constante de temps d'environ 3 minutes.

Le principe du Réglage Secondaire de la tension (RST) consiste à organiser le réseau en «zones » de réglage et à réguler la tension d'un point particulier de chaque zone, appelé «point pilote» (cf. [5]). Un calculateur centralisé situé dans le dispatching régional élabore pour chaque zone de réglage un signal de commande $\mathrm{N}_{\mathrm{RST}}$, appelé niveau, pouvant varier entre -1 et 1 . Pour chaque zone, le niveau correspondant est envoyé à l'ensemble des groupes réglants de la zone et est transformé localement en variations de tension de consigne appliquées au régulateur primaire de tension. La Figure 1 explicite le fonctionnement de ce réglage.

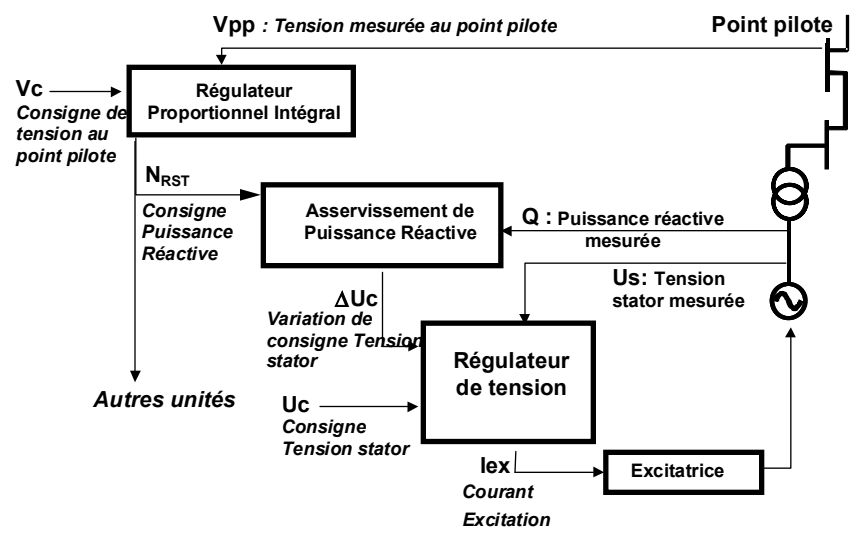

Figure 1. Réglage Secondaire de la Tension

Une version améliorée du réglage secondaire de tension, le RSCT (Réglage Secondaire Coordonné de Tension), a été développée et mise en service dans la région Ouest particulièrement sensible aux problèmes de tensions. ([5], [6], [7]).

\section{Caracteristiques Du Reglage De la Frequence}

L'évolution de la fréquence est l'image directe du déséquilibre entre la production et la consommation. Une augmentation de la fréquence signifie que le bilan production-consommation est excédentaire ; une diminution que ce même bilan est déficitaire. Ceci est valable à l'échelle du réseau européen puisque les différents réseaux électriques qui le composent sont interconnectés par des liaisons synchrones. L'ensemble des charges et des groupes de production européens forment donc un même réseau de fréquence uniforme.

Face aux évolutions normales de la consommation et aux divers aléas rencontrés en exploitation (déclenchements d'installations de production ou de charges, etc.) le maintien de l'équilibre production-consommation et le maintien d'une valeur satisfaisante de la fréquence nécessitent d'adapter en permanence le niveau de la production à celui de la demande.

Pour réaliser cette adaptation de la production, il faut disposer de réserves de puissance mobilisables automatiquement (réglages primaire et secondaire) ou par l'action des opérateurs (réglage tertiaire).

Dans ce sens, RTE, en tant que membre de l'UCTE, s'engage à respecter les principes de réglage de la fréquence explicités dans 1'UCTE Operational Handbook, [8]. 


\section{A. Réglage Primaire de Fréquence}

Ce réglage permet de rétablir automatiquement et en quelques secondes l'équilibre production-consommation et la fréquence à une valeur proche de la fréquence de référence ( $50 \mathrm{~Hz}$ en général). Le réglage de fréquence est mis en œuvre par l'action des régulateurs de vitesse des groupes de production qui agissent en général sur les organes d'admission du fluide moteur à la turbine lorsque la vitesse du groupe, image de la fréquence du réseau, s'écarte de la vitesse de consigne suite à un déséquilibre productionconsommation sur le système électrique interconnecté.

Une unité de production participant au réglage primaire et disposant de réserve «primaire» mobilisable par cet automatisme, doit produire :

$\mathrm{P}(\mathrm{t})=\mathrm{P}_{0}+\mathrm{K}$

où $\mathrm{P}(\mathrm{t})$ est la puissance active produite à l'instant $\mathrm{t}$ et $\Delta \mathrm{f}(\mathrm{t})$ l'écart de fréquence au même instant. $\mathrm{P}_{0}$ est le programme de marche de l'installation et $\mathrm{K}$ son gain de réglage primaire exprimé en $\mathrm{MW} / \mathrm{Hz}$.

\section{B. Réglage Secondaire Fréquence/Puissance}

L'action du réglage primaire laisse subsister un écart de fréquence par rapport à la fréquence de référence. Elle provoque également des écarts sur les échanges entre les pays du système électrique interconnecté.

Le système électrique européen est organisé en zones de réglage. Le réglage secondaire a pour objectif de ramener en moins de quinze minutes la fréquence à sa valeur de référence et les échanges entre pays à leur valeur contractuelle, restituant ainsi l'intégralité de la réserve primaire engagée par les membres de l'interconnexion européenne. Cette restitution permet de pouvoir pallier rapidement tout nouveau déséquilibre productionconsommation.

Le réglage secondaire est réalisé par un organe centralisé situé au centre de conduite de la zone de réglage et dont le rôle est de modifier automatiquement le programme de production des centrales jusqu'à annuler l'écart de réglage de la zone. Pour cela, ce régulateur centralisé élabore, à partir des télémesures de la fréquence et des transits sur les lignes d'interconnexion, un signal $\mathrm{N}$ appelé niveau de téléréglage, compris entre -1 et +1 et l'envoie aux groupes de production afin de modifier leurs puissances de consigne.

Une unité de production participant aux réglages primaire et secondaire doit produire :

$$
\mathrm{P}(\mathrm{t})=\mathrm{P}_{0}+\mathrm{K} \cdot \Delta \mathrm{f}(\mathrm{t})+\mathrm{N}(\mathrm{t}) \cdot \mathrm{P}_{\mathrm{r}}
$$

où $\mathrm{P}(\mathrm{t})$ est la puissance active produite à l'instant $\mathrm{t}, \Delta \mathrm{f}(\mathrm{t})$ l'écart de fréquence au même instant et $N(t)$ la valeur du niveau. $\mathrm{P}_{0}$ est le programme de marche de l'installation, $\mathrm{K}$ son gain primaire exprimé en $\mathrm{MW} / \mathrm{Hz}$ et $\mathrm{P}_{\mathrm{r}}$ son engagement de participation au réglage secondaire, en $\mathrm{MW}$.

Par ailleurs, les groupes de production ont des temps de réponse variables en fonction de leur technologie et des réglages de leurs régulations. Il est donc important de s'assurer que la réaction des réglages primaire et secondaire de l'unité participant au réglage de la fréquence est suffisamment rapide pour que l'équilibre productionconsommation soit rétabli promptement et sans instabilité.

\section{Le Controle des Performances}

Comme indiqué dans le paragraphe II, RTE a mis en place récemment un mécanisme de contrôle des performances en matière de réglage de fréquence et de tension. En effet, compte-tenu de l'importance de ces performances pour l'exploitation et la sûreté du système électrique, il est fondamental pour un gestionnaire de réseau de pouvoir s'assurer de la fourniture effective de ces réglages et d'être alerté sur les éventuels dysfonctionnements.

Le contrôle des performances actuellement mis en œuvre est fondé sur un outil dont les caractéristiques principales sont décrites ci-après. Nous présentons également des exemples d'analyses réalisées avec ce logiciel.

\section{A. L'outil CDP Prod}

L'outil CDP Prod analyse les mesures de fréquence, de puissance active et réactive et de tension en sortie de générateur, calcule des indicateurs et fournit ainsi des éléments de diagnostic a posteriori en $\mathrm{J}+1$ permettant d'apprécier qualitativement et quantitativement la contribution d"une installation de production aux Services Système.

L'outil exploite diverses informations en provenance du système d'information de RTE : programmation, conduite, comptage et n'a donc pas nécessité l'installation d'équipements de mesure spécifiques.

En ce qui concerne le réglage de tension et le réactif, deux types d'analyse sont effectués à ce jour pour chaque installation :

- Le suivi continu des points de fonctionnement de l'installation de production dans le diagramme [U/Q] contractualisé avec le producteur

- La participation correcte au réglage secondaire de tension d'un point de vue statique et dynamique

Pour le réglage de la fréquence, les analyses suivantes sont réalisées pour chaque installation :

- Le suivi continu du gain primaire $\mathrm{K}$ et de la participation $\mathrm{P}_{\mathrm{r}}$ au réglage secondaire ainsi que l'estimation des réserves de puissance de l'installation de production

- La performance dynamique des réglages primaire et secondaire. 


\section{B. Quelques exemples d'analyse}

1) Contrôle de la disponibilité du diagramme [U/Q] contractualisé

Cette performance est vérifiée en traçant les points de fonctionnement de l'alternateur dans le plan [U/Q] de manière continue. Lorsque l'alternateur est exploité aux limites du diagramme contractualisé $[\mathrm{U} / \mathrm{Q}]$ du fait de contraintes de tension régulières ou occasionnelles, on peut vérifier la valeur maximale de puissance réactive qui peut être générée par l'installation. La Figure 2. ci-dessous illustre les conditions d'exploitation d'un alternateur (Q stator en abscisse, U stator en ordonnée) pendant le mois de mars 2005. Pour cette installation de production, la puissance réactive maximale générée a été obtenue dès que la tension stator a atteint 1.05 p.u., conformément à ce qui a été contractualisé avec RTE.
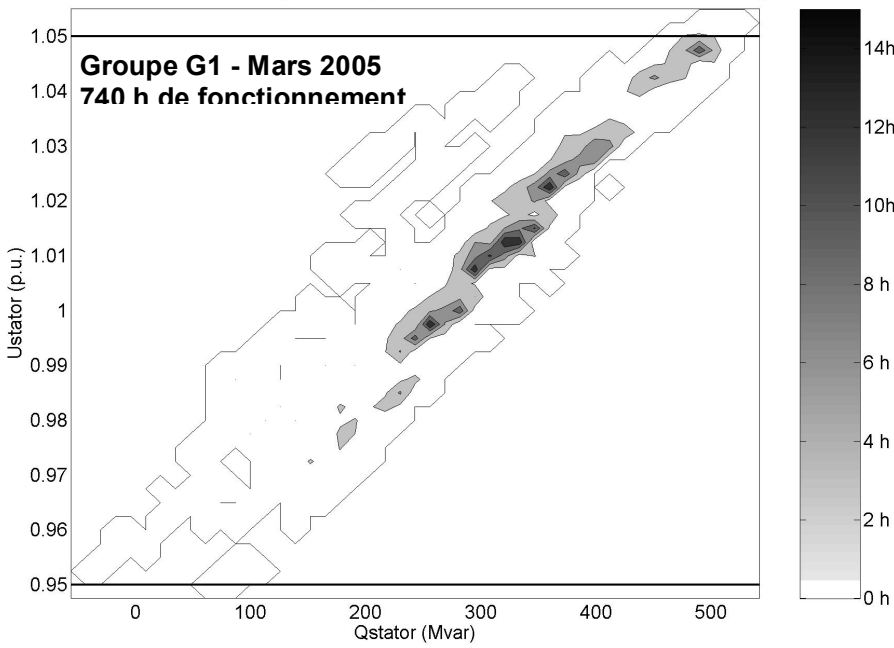

Figure 2. Domaine [U/Q] du groupe G1 en Mars 2005

2) Contrôle de la participation au Réglage Secondaire de Tension

a) Performance statique

Comme évoqué au paragraphe III, la puissance réactive d'un groupe réglant doit être proportionnelle au niveau $\mathrm{N}_{\mathrm{RST}}$ transmis par le dispatching régional, selon la loi statique suivante :

$$
\mathrm{Q}_{\mathrm{st}}=\mathrm{N}_{\mathrm{RST}} \cdot \mathrm{Q}_{\mathrm{r}}
$$

où $\mathrm{Q}_{\mathrm{r}}$ est l'engagement contractuel de l'unité de production de participation au Réglage Secondaire de Tension.

L'outil calcule de manière continue l'erreur absolue $\varepsilon$ entre la puissance réactive réellement produite par le groupe de production et le comportement du groupe :

$$
\varepsilon=\left|\mathrm{Q}_{\mathrm{st}} / \mathrm{Q}_{\mathrm{r}}-\mathrm{N}_{\mathrm{RST}}\right|
$$

La participation de l'installation au Réglage Secondaire de Tension est considérée comme satisfaisante tant que $\varepsilon$ est plus petit qu'un seuil de tolérance pendant une durée significative. La Figure 3. illustre le principe de contrôle de la performance du Réglage Secondaire de Tension.
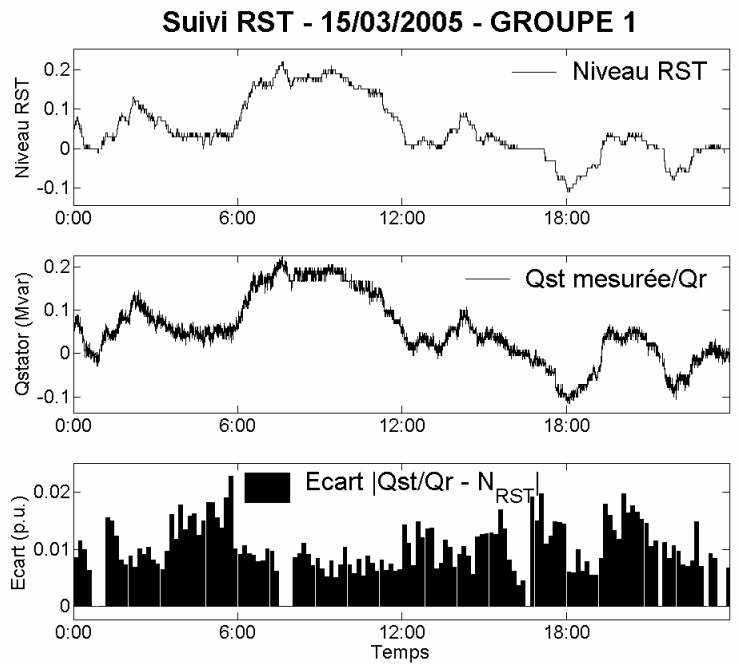

Figure 3. Contrôle du Réglage Secondaire de Tension

\section{b) Performance dynamique}

Afin d'assurer un temps de réponse global suffisant pour le Réglage Secondaire de Tension, le temps de réponse de la boucle locale, au niveau de chaque installation de production, ne doit pas excéder 60s. Ce temps de réponse est évalué en estimant le retard entre la mesure de la puissance réactive et la consigne $\mathrm{N}_{\mathrm{RST}}$. Qr définie en (3). Cette estimation est plus facile à réaliser pendant les périodes où le niveau $\mathrm{N}_{\mathrm{RST}}$ varie en rampe, comme illustré dans l'exemple suivant :
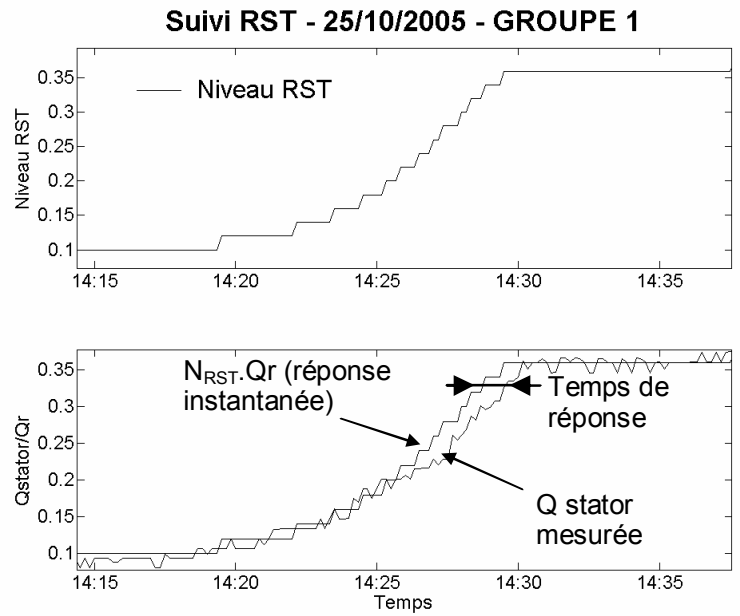

Figure 4. Illustration du principe de contrôle du temps de réponse d'un groupe au réglage secondaire de la tension

Dans cet exemple, on peut considérer que le temps de réponse de la boucle locale est satisfaisant. 


\section{3) Contrôle de la participation au Réglage de} Fréquence

\section{a) Performance statique}

Une unité de production participant aux réglages primaire et secondaire doit produire :

$$
\mathrm{P}(\mathrm{t})=\mathrm{P}_{0}+\mathrm{K} \cdot \Delta \mathrm{f}(\mathrm{t})+\mathrm{N}(\mathrm{t}) \cdot \mathrm{P}_{\mathrm{r}}
$$

CDP Prod estime par pas de 10 minutes les paramètres de réglage du groupe $P_{0}, K$ et $P_{r}$. L'estimation est réalisée par la méthode des moindres carrés appliqués aux réalisations de l'équation (2), disponibles avec une périodicité de 10 secondes.

Le groupe est considéré en écart lorsque les paramètres estimés sont inférieurs aux valeurs attendues compte-tenu des engagements du producteur.

Le même principe est appliqué à l'équation (1) pour les groupes qui ne participent qu'au réglage primaire de la fréquence.

La Figure 5. présente les résultats obtenus pour un groupe sur la première quinzaine de décembre 2005. Pour les paramètres $\mathrm{K}$ et $\mathrm{P}_{\mathrm{r}}$, la figure superpose en fonction du temps les valeurs contractuellement programmées (trait fin continu) et les valeurs estimées au pas de dix minutes (trait épais discontinu). Ce type de représentation permet de vérifier si à un instant donné le groupe a été hors réglage alors que la programmation l'engageait à participer aux Services Système de réglage de la fréquence.
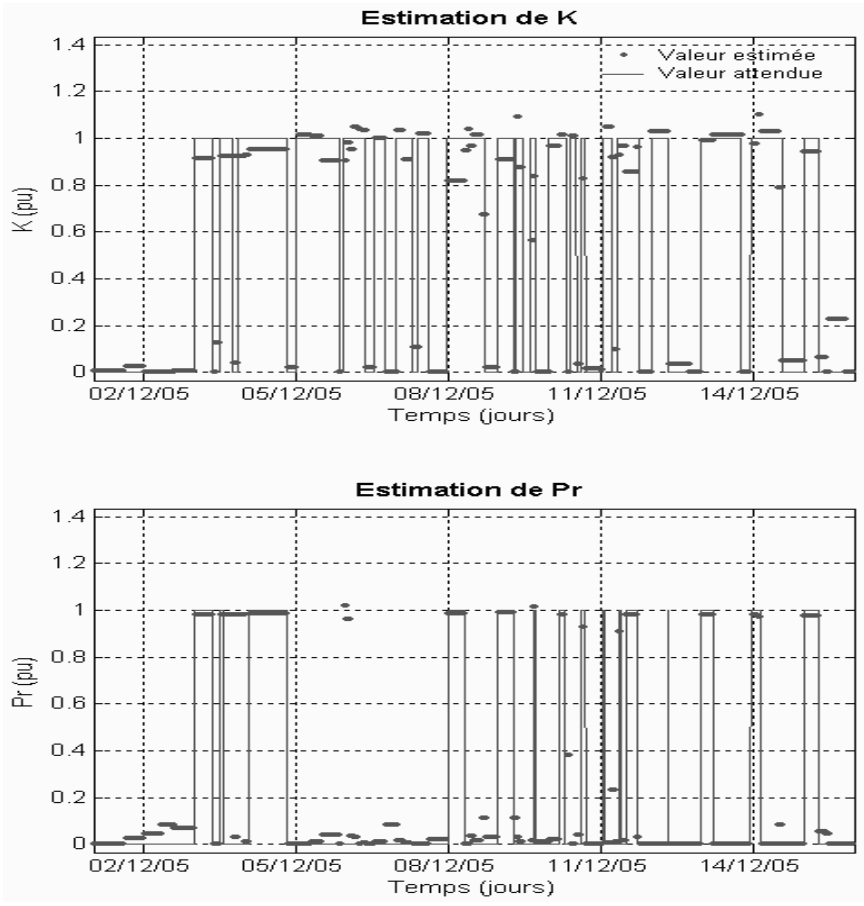

Figure 5. Exemple de contrôle pour un groupe sur la période du $1 \mathrm{er}$ au 15/12/2005 - Paramètres K et Pr en fonction du temps
La Figure 6. présente les distributions des valeurs estimées de $\mathrm{K}$ et $\mathrm{P}_{\mathrm{r}}$ de la figure précédente. Sur des périodes suffisamment longues, cette représentation permet de vérifier statistiquement le réglage réel du paramètre au niveau du groupe. Dans le cas présent, les valeurs de $\mathrm{K}$ et $\mathrm{P}_{\mathrm{r}}$ sont bien distribuées autour de 1 p.u., donc correctement réglées pour ce groupe.
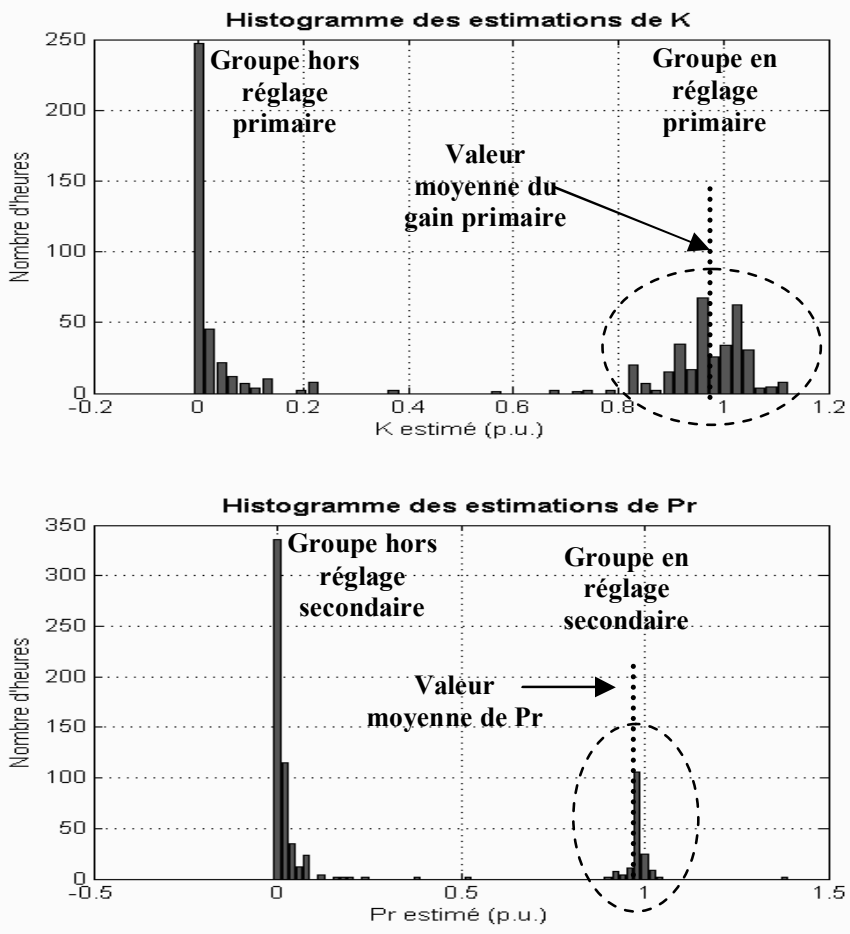

Figure 6. Exemple de contrôle pour un groupe du 1er au 15/12/2005 - Distribution statistique des paramètres K et Pr

Dès lors que les statistiques établies à l'aide de l'estimation des paramètres $\mathrm{K}$ et $\operatorname{Pr}$ mettent en évidence un écart, celui-ci est systématiquement illustré par des éléments concrets, en comparant la réponse mesurée du groupe et la réponse contractuellement attendue compte-tenu des engagements du producteur. Ce type de comparaison est illustré par la Figure 7.

\section{b) Performance dynamique}

De même que pour le temps de réponse au réglage secondaire de tension, le temps de réponse aux réglages primaire et secondaire de fréquence est évalué.

Pour le réglage primaire, on examine la variation de puissance du groupe suite à une variation rapide de fréquence (lors d'une perte importante de production par exemple). La qualité de la réponse dynamique du groupe est suffisante si la réponse du groupe est plus rapide qu'un gabarit minimum acceptable par RTE.

Pour le réglage secondaire, le temps de réponse au réglage secondaire $\mathrm{F} / \mathrm{P}$ est évalué par une méthode similaire à celle présentée pour le RST, lors de variations en rampe du niveau de téléréglage. 


\section{Suivi Continu FIP - 02/03/2005 - Groupe G2}
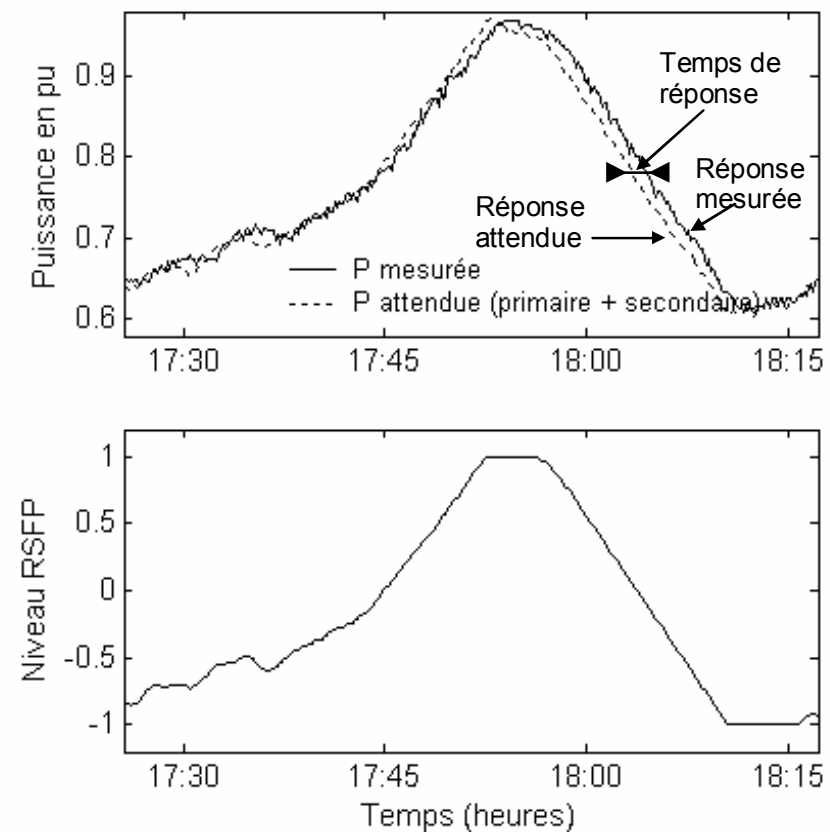

Figure 7. Illustration du principe de contrôle du temps de réponse d'un groupe au réglage secondaire de la fréquence

\section{CONCLUSION}

La disponibilité des capacités de réglage et la performance des régulateurs des groupes de production constitue un enjeu technique et financier important pour un gestionnaire de réseau. C'est la raison pour laquelle RTE a complété le dispositif de prescription au raccordement de toute nouvelle unité de production et de contractualisation de la fourniture des Services Système en exploitation, par la mise en place d'un mécanisme de contrôle des performances.

Ce mécanisme, fondé sur des mesures adaptées et graduelles ainsi que sur l'utilisation d'un outil dédié, a été mis en œuvre à partir de 2005. Les modalités de contrôle sont précisées dans les contrats de Service Système signés par les producteurs.

La mise en place du contrôle des performances a également contribué à créer un dialogue continu entre RTE et les producteurs sur les performances techniques des centrales, à améliorer la connaissance des performances des groupes de production et à mieux définir les responsabilités de chacun des acteurs grâce aux travaux de spécification qui ont été réalisés dans ce cadre.

\section{REFERENCES}

[1] U.S. - Canada Power System Outage Task Force "Final Report on the August 14, 2003 Blackout in the United States and Canada. Causes and Recommendations", April 2004

[2] Ministère de l'économie, des finances et de l'industrie, "Décret $n^{\circ}$ 2003-588 du 27 juin 2003 relatif aux prescriptions techniques générales de conception et de fonctionnement auxquelles doivent satisfaire les installations en vue de leur raccordement au réseau public de transport d'électricité", [J.O. n 151, 2 juillet 2003, pp. 11110-11113]

[3] Ministère de l'économie, des finances et de l'industrie, "Arrêté du 4 juillet 2003 relatif aux prescriptions techniques de conception et de fonctionnement pour le raccordement au réseau public de transport d'une installation de production d'énergie électrique", [J.O. n ${ }^{\circ} 201$, 31 août 2003, pp. 14896-14902]

[4] Référentiel technique de RTE, http:www.rte-France.com

[5] J.P. Paul, C. Corroyer, P. Jeannel, J.M. Tesseron, F. Maury, A. Torra, "Improvements in the organization of secondary voltage control in France", CIGRE, PARIS, 1990, rapport 38/39-03

[6] P. Panciatici, F. Bena, P. Pruvost, N. Janssens J. Deuse, M. Stubbe "Centralized voltage control: a key point for optimal operation of power systems", CIGRE, PARIS, 1998, rapport 39-116

[7] H. Lefebvre, D. Fragnier, J.Y. Boussion, P. Mallet, M. Bulot, "Secondary Coordinated Voltage Control System : Feedback of EDF", IEEE Summer Meeting, SEATTLE, July 2000

[8] UCTE Operation Handbook - Policy 1 "Load-Frequency Control and Performance" et Appendix 1 "Load-Frequency Control and Performance", http://www.ucte.org 